\title{
Características etiológicas, clínicas y sociodemográficas de la diarrea aguda en Venezuela
}

\author{
María I. Urrestarazu, ${ }^{1}$ Ferdinando Liprandi, ${ }^{2}$ Eva Pérez de Suárez, ${ }^{3}$ \\ Rosabel González ${ }^{4}$ e Irene Pérez-Schael ${ }^{4}$
}

RESUMEN En cuatro ciudades de Venezuela se llevó a cabo un estudio para evaluar las características epidemiológicas, clínicas y etiológicas de la diarrea aguda en niños menores de 5 años. Entre junio de 1993 y mayo de 1995 se estudiaron 252 niños con diarrea y 793 controles que fueron atendidos en el hospital. Para el análisis estadístico de los resultados se empleó la prueba exacta de Fisher. Los rotavirus fueron los agentes más importantes, tanto por su frecuencia (30\%) como por su asociación con la deshidratación (58\%). Le siguieron en importancia Campylobacter spp. (13\%) y Escherichia coli serogrupos O clásicos (9\%), pero su asociación con la diarrea solo fue estadisticamente significativa en los niños menores de 3 meses, hecho de particular importancia desde el punto de vista del tratamiento. Se confirmó la importancia de la edad como factor determinante de la prevalencia y gravedad de la diarrea.

Las enfermedades diarreicas constituyen un problema de salud pública en el mundo, especialmente en los países en desarrollo, donde representan una importante causa de morbilidad y mortalidad en niños menores de 5 años. Múltiples episodios de diarrea

\footnotetext{
1 Sección de Microbiología, Instituto de Biomedicina, Universidad Central de Venezuela, Ministerio de Sanidad y Asistencia Social, Caracas, Venezuela. La correspondencia debe dirigirse a María Isabel Urrestarazu a la siguiente dirección postal: Instituto de Biomedicina, Sección de Microbiología, Apartado Postal 4043, Caracas 1010 A, Venezuela. Fax: 00-58-2-8611258.

2 Laboratorio de Biología de Virus, Centro de Microbiología, Instituto Venezolano de Investigaciones Científicas, Altos de Pipe, Venezuela.

3 Laboratorio de Amebiasis, Cátedra de Parasitología, Escuela de Bioanálisis, Facultad de Medicina, Universidad Central de Venezuela, Caracas, Venezuela.

4 Sección de Investigación de Enfermedades Entéricas, Instituto de Biomedicina, Universidad Central de Venezuela, Ministerio de Sanidad y Asistencia Social, Fuvesin, Caracas, Venezuela.
}

en el primer año de vida pueden deteriorar el estado nutricional y causar graves secuelas $(1,2)$. Se ha estimado que, en Asia, África y América Latina, cada año mueren alrededor de 3,3 millones de niños menores de 5 años por diarrea (3) y ocurren más de mil millones de episodios (4). En Venezuela, se ha estimado (5) que ocurren 1,32 millones de episodios anuales de diarrea, con una mediana de 2,2 episodios por niño y año, cifra muy similar a la registrada (3) en todo el mundo: 2,5 episodios por niño y año. En los últimos años, las diarreas han representado en Venezuela la novena causa de muerte en la población en general y la segunda causa de mortalidad en menores de 4 años (6). De los 681928 casos de diarrea registrados en 1998, 30\% ocurrieron en menores de 1 año (7).

Las características epidemiológicas, agentes etiológicos y presentación clí- nica de las diarreas son muy variables dependiendo del país, región y comunidad, por lo que su conocimiento es esencial para el diseño de programas de prevención y control, como, por ejemplo, la introducción de vacunas frente a los rotavirus (8-10).

El objetivo de este trabajo consistió en investigar la epidemiología (edad, sexo, distribución geográfica, condiciones socioeconómicas y nutricionales), causas y manifestaciones clínicas de la diarrea aguda en niños menores de 5 años en cuatro ciudades de Venezuela.

\section{MATERIALES Y MÉTODOS}

\section{Población de estudio}

Entre junio de 1993 y mayo de 1995 se estudiaron 2552 casos de diarrea 
aguda y 793 controles en cuatro ciudades de Venezuela (cuadro 1).

Los criterios de inclusión de los casos fueron los siguientes: edad inferior a 5 años, diarrea aguda de evolución no superior a 72 horas, no haber recibido antibióticos durante las tres semanas anteriores al episodio, no encontrarse en estado de choque ni gravemente desnutrido y no padecer alteraciones congénitas. Se incluyeron todos los casos que cumplían estos criterios y que consultaron entre las 7.00 y las 13.00 horas. A su vez, los criterios de inclusión de los controles consistieron en: edad inferior a 5 años y no haber recibido antibióticos ni haber padecido episodios de diarrea durante las 6 semanas anteriores a la entrevista. Los controles fueron seleccionados en las consultas ambulatorias o en las salas de hospitalización. Un episodio de diarrea aguda se definió como la presencia de tres evacuaciones líquidas o semilíquidas, o de una evacuación de consistencia alterada y sanguinolenta, en 24 horas.

Después de informar al representante del niño de los objetivos y características del estudio y de obtener su consentimiento, se registraron los siguientes datos en el momento de la consulta: edad, sexo, procedencia, nivel socioeconómico según el método de Graffar modificado (11) y, en los niños de 0 a 3 meses de edad, el tipo de alimentación (materna, artificial o mixta). En la historia clínica se anotaron las manifestaciones clínicas (fiebre, vómitos, número de evacuaciones, deshidratación y duración de la diarrea) y las características de las evacuaciones (consistencia y presencia de sangre) hasta la curación del episodio.

\section{Procesamiento de las muestras}

La presencia de rotavirus se investigó en muestras de heces conservadas a $-20{ }^{\circ} \mathrm{C}$ mediante un inmunoensayo enzimático (ELISA) pre/post, que permite identificar los positivos falsos gracias a la utilización de un control para reacciones inespecíficas (12). En un subgrupo de 377 muestras procedentes de Caracas se identificaron los serotipos $G$ mediante ELISA utilizando anticuerpos monoclonales específicos $(13,14)$.

Para la investigación de bacterias, las muestras fueron tomadas mediante hisopado rectal, colocadas en medio de transporte de Cary Blair y mantenidas a $4{ }^{\circ} \mathrm{C}$ hasta su procesamiento, realizado en un tiempo no mayor de 48 horas. Las muestras fueron sembradas en agar de MacConkey, agar Salmonella-Shigella, agar de xilosa-lisina-desoxicolato (XLD), caldo Selenito y, para la investigación de Campylobacter, en agar sin sangre suplementado con cefoperazona. Los microorganismos aislados se identificaron por métodos estandarizados (15).

En una submuestra de la población (513 pacientes y 241 controles), se aislaron cinco colonias de Escherichia coli para determinar sus características patógenas. El serogrupo se determinó por el método de aglutinación en lámina con antisueros polivalentes $\mathrm{y}$ monovalentes contra antígenos $\mathrm{O}$ de E. coli (Denka-Seiken Co. Ltd., Japón) y los positivos se confirmaron por aglutinación en tubo mediante procedimientos convencionales (16). También se investigó la producción de toxina termolábil mediante aglutinación de látex pasiva inversa (reversed passive latex agglutination, VET-RPLA, DenkaSeiken Co. Ltd., Japón) y de toxina termoestable mediante ELISA (COLIST EIA, Denka-Seiken Co. Ltd., Japón) $(17,18)$. La capacidad invasora se determinó en cultivo de células HEp 2 según el método de Day et al. (19).

Los parásitos Entamoeba histolytica, Blastocystis hominis y Giardia lamblia se investigaron en la hora siguiente a la obtención de la muestra, mediante examen en fresco de las heces por observación directa con solución salina a $0,85 \%$ y con las coloraciones de Quensel, Lugol y Sudán III. En un subgrupo de niños se confirmó la presencia de Entamoeba histolytica y Blastocystis hominis utilizando la coloración de hematoxilina férrica y el cultivo de las heces en medio de Boeck y Drbohlav (20). Para visualizar Cryptosporidium paroum se realizó un extendido teñido con la coloración de Ziehl-Neelsen.

\section{Análisis estadístico}

Se utilizó la prueba exacta de Fisher.

\section{RESULTADOS}

\section{Características de la población estudiada}

Aunque los niños predominaron sobre las niñas en ambos grupos, la proporción de varones fue significativamente mayor en los casos (cuadro 2). Los dos grupos no presentaron diferencias significativas en relación con la edad ni el nivel socioeconómico. La media de la edad fue idéntica en ambos grupos (6 meses). La distribu-

CUADRO 1. Procedencia de los individuos estudiados. Venezuela, 1993-1995

\begin{tabular}{|c|c|c|c|c|c|c|}
\hline \multirow[b]{2}{*}{ Ciudad } & \multicolumn{2}{|c|}{$\begin{array}{l}\text { Pacientes } \\
(n=2552)\end{array}$} & \multicolumn{2}{|c|}{$\begin{array}{l}\text { Controles } \\
(n=793)\end{array}$} & \multirow[b]{2}{*}{ Centro hospitalario } & \multirow{2}{*}{$\begin{array}{c}\text { Camas } \\
\text { pediátricas }\end{array}$} \\
\hline & No. & $\%$ & No. & $\%$ & & \\
\hline Caracas & 1285 & 50 & 429 & 54 & $\begin{array}{l}\text { Centro Maternoinfantil "Dr. Pastor Oropeza" } \\
\text { Hospital de Niños "J. M. de los Ríos" }\end{array}$ & $\begin{array}{r}95 \\
500\end{array}$ \\
\hline Cumaná & 430 & 17 & 94 & 12 & Hospital Universitario "Antonio Patricio Alcalá" & 75 \\
\hline Mérida & 461 & 18 & 148 & 19 & Hospital Universitario "Los Andes" & 70 \\
\hline Puerto Ordaz & 376 & 15 & 122 & 15 & Hospital Pediátrico "Menca de Leoni" & 102 \\
\hline
\end{tabular}


ción de los pacientes por grupos de edad fue la siguiente: 589 (23\%) de 0 a 2 meses, $552(22 \%)$ de 3 a 5 meses, 433 $(17 \%)$ de 6 a 8 meses, $315(12 \%)$ de 9 a 11 meses y $633(26 \%)$ de 12 meses o más. La distribución en el grupo de control fue: 229 (29\%) de 0 a 2 meses, $134(17 \%)$ de 3 a 5 meses, $109(14 \%)$ de 6 a 8 meses, $60(8 \%)$ de 9 a 11 meses y $261(33 \%)$ de 12 meses o más. Los menores de 1 año representaron $74 \%$ de los casos y $67 \%$ de los controles. Tanto en un grupo como en el otro, la mayoría de los niños pertenecieron a las clases socioeconómicas más desfavorecidas (estratos 4 y 5 de Graffar) (cuadro 2). Mérida presentó un comportamiento diferente al de las demás ciudades, con menor proporción de casos menores de 1 año (58\%) y mayor proporción de casos en los estratos sociales más altos $(16 \%$, frente a $1 \%$ en Caracas, $3 \%$ en Cumaná y $1 \%$ en Puerto Ordaz; $P<0,001$ ).

La desnutrición fue significativamente más frecuente en los niños con diarrea, mientras que la lactancia materna durante los 3 primeros meses de vida fue significativamente más frecuente en los controles (cuadro 2). Mérida presentó la mayor proporción de casos con lactancia exclusivamente materna $(47 \%$, frente a $16 \%$ en Caracas, $27 \%$ en Cumaná, y $14 \%$ en Puerto Ordaz; las diferencias con Caracas y Puerto Ordaz fueron estadísticamente significativas: $P<0,05)$ y una frecuencia de desnutrición (16\%) inferior a la de Cumaná $(28 \% ; P<0,001)$ y Puerto Ordaz (35\%; $P<0,001)$, pero superior a la de Caracas, $(9 \% ; P<0,001)$. El tratamiento de la diarrea se realizó en régimen ambulatorio en $46 \%$ de los casos; el resto necesitó hospitalización.

\section{Frecuencia de los agentes etiológicos}

En una elevada proporción de casos (41\%) no se identificó ningún agente patógeno. Los agentes etiológicos detectados con mayor frecuencia en los niños con diarrea fueron los rotavirus $(30 \%)$, seguidos de Campylobacter spp. $(13 \%)$, E. coli de los serogrupos O clásicos (9\%), Shigella spp. (7\%) y E. coli enterotoxígena $(5 \%)$; todos estos micro-

CUADRO 2. Características de la población estudiada. Venezuela, 1993-1995

\begin{tabular}{|c|c|c|c|c|c|}
\hline \multirow[b]{2}{*}{ Características } & \multicolumn{2}{|c|}{$\begin{array}{l}\text { Pacientes } \\
(n=2552)\end{array}$} & \multicolumn{2}{|c|}{$\begin{array}{l}\text { Controles } \\
(n=793)\end{array}$} & \multirow[b]{2}{*}{$P^{a}$} \\
\hline & No. & $\%$ & No. & $\%$ & \\
\hline Masculino/Femenino & $1502 / 1050$ & $59 / 41$ & $427 / 366$ & $54 / 46$ & $<0,05$ \\
\hline \multicolumn{6}{|l|}{ Estrato socioeconómico } \\
\hline 1-2 (clase alta + media) & 108 & 4 & 29 & 4 & $\mathrm{NS}^{\mathrm{b}}$ \\
\hline 3 (clase media baja) & 499 & 20 & 175 & 22 & NS \\
\hline 4-5 (clase baja + marginal) & 1945 & 76 & 589 & 74 & NS \\
\hline \multicolumn{6}{|l|}{ Estado nutricional } \\
\hline Eutrófico & 2099 & 82 & 736 & 93 & $<0,001$ \\
\hline Desnutrido & 453 & 18 & 57 & 7 & - \\
\hline \multicolumn{6}{|l|}{ Tipo de alimentaciónc } \\
\hline Lactancia materna & 168 & 21 & 113 & 38 & $<0,001$ \\
\hline Lactancia mixta & 312 & 39 & 109 & 37 & NS \\
\hline Lactancia artificial & 315 & 40 & 74 & 25 & $<0,001$ \\
\hline
\end{tabular}

a Prueba exacta de Fisher.

${ }^{\mathrm{b}}$ No significativo.

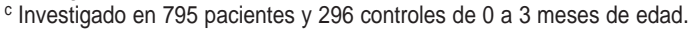

organismos fueron significativamente más frecuentes en los casos que en los controles. Los parásitos no desempeñaron un papel importante como causa de diarrea: Blastocystis hominis, Entamoeba histolytica y Cryptosporidium spp. se encontraron con igual frecuencia en ambos grupos, mientras que
Giardia lamblia fue significativamente más frecuente en los controles que en los pacientes (cuadro 3 ).

Casi un tercio $(29 \%)$ de los rotavirus no pudieron ser tipificados por ELISA. El serotipo predominante fue el G1 (70\%), y el G2 solo se detectó de forma muy esporádica ( $1 \%)$.

CUADRO 3. Frecuencia de los agentes etiológicos de diarrea. Venezuela 1993-1995

\begin{tabular}{|c|c|c|c|c|c|}
\hline \multirow[b]{2}{*}{ Agente etiológico } & \multicolumn{2}{|c|}{ Pacientes } & \multicolumn{2}{|c|}{ Controles } & \multirow[b]{2}{*}{$P^{a}$} \\
\hline & No. & $\%$ & No. & $\%$ & \\
\hline Rotavirus $^{b}$ & 693 & 30 & 25 & 4 & $<0,001$ \\
\hline $\begin{array}{l}\text { Enteropatógenos }{ }^{\mathrm{C}} \\
\text { Campylobacter spp. } \\
\text { Shigella spp. } \\
\text { Salmonella spp. } \\
\text { Aeromonas spp. }\end{array}$ & $\begin{array}{r}315 \\
169 \\
51 \\
54\end{array}$ & $\begin{array}{r}13 \\
7 \\
2 \\
2\end{array}$ & $\begin{array}{r}71 \\
14 \\
7 \\
14\end{array}$ & $\begin{array}{l}9 \\
2 \\
1 \\
2\end{array}$ & $\begin{array}{c}0,01 \\
<0,001 \\
\text { NS } \\
\text { NS }\end{array}$ \\
\hline $\begin{array}{l}\text { E. colid } \\
\text { Serogrupos O clásicos } \\
\text { Enterotoxígena } \\
\text { Enteroinvasora } \\
\text { Enterohemorrágica }\end{array}$ & $\begin{array}{r}47 \\
23 \\
2 \\
0\end{array}$ & $\begin{array}{r}9 \\
5 \\
<1 \\
0\end{array}$ & $\begin{array}{l}1 \\
0 \\
0 \\
0\end{array}$ & $\begin{array}{r}<1 \\
0 \\
0 \\
0\end{array}$ & $\begin{array}{c}<0,001 \\
<0,001 \\
\text { NS } \\
?\end{array}$ \\
\hline $\begin{array}{l}\text { Parásitos }^{\mathrm{e}} \\
\text { Giardia lamblia } \\
\text { Blastocystis hominis } \\
\text { Entamoeba histolytica } \\
\text { Cryptosporidium spp. }\end{array}$ & $\begin{array}{l}45 \\
88 \\
19 \\
16\end{array}$ & $\begin{array}{l}2 \\
4 \\
1 \\
1\end{array}$ & $\begin{array}{r}33 \\
27 \\
0 \\
0\end{array}$ & $\begin{array}{l}6 \\
4 \\
0 \\
0\end{array}$ & $\begin{array}{c}<0,001 \\
\text { NS } \\
\text { NS } \\
\text { NS }\end{array}$ \\
\hline Sin agente patógeno & 1031 & 41 & 423 & 70 & $?$ \\
\hline
\end{tabular}

a Prueba exacta de Fisher.

b Investigados en una submuestra aleatoria de 2347 pacientes y 601 controles.

c Investigados en una submuestra aleatoria de 2515 pacientes y 781 controles.

d Investigada en una submuestra aleatoria de 513 pacientes y 241 controles.

e Investigados en una submuestra aleatoria de 2334 pacientes y 577 controles. 
CUADRO 4. Distribución de los patógenos según la edad. Venezuela 1993-1995

\begin{tabular}{|c|c|c|c|c|c|c|c|c|c|c|c|c|}
\hline \multirow[b]{3}{*}{ Agentes patógenos } & \multicolumn{12}{|c|}{ Porcentajes de pacientes $(\mathrm{P})$ y controles $(\mathrm{C})$ según grupos de edad (meses) } \\
\hline & \multicolumn{2}{|c|}{$0-2$} & \multicolumn{2}{|c|}{$3-5$} & \multicolumn{2}{|c|}{$6-8$} & \multicolumn{2}{|c|}{$9-11$} & \multicolumn{2}{|c|}{$12-23$} & \multicolumn{2}{|c|}{$\geq 24$} \\
\hline & $P$ & C & $\mathrm{P}$ & C & $\mathrm{P}$ & C & $\mathrm{P}$ & C & $\mathrm{P}$ & C & $\mathrm{P}$ & C \\
\hline $\begin{array}{l}\text { No. de individuos } \\
\text { Rotavirus (\%) }\end{array}$ & $\begin{array}{c}540 \\
16^{\mathrm{a}}\end{array}$ & $\begin{array}{r}172 \\
3\end{array}$ & $\begin{array}{r}487 \\
39^{a}\end{array}$ & $\begin{array}{r}112 \\
4\end{array}$ & $\begin{array}{r}415 \\
34^{\mathrm{a}}\end{array}$ & $\begin{array}{r}67 \\
1\end{array}$ & $\begin{array}{c}310 \\
37^{a}\end{array}$ & $\begin{array}{r}59 \\
8\end{array}$ & $\begin{array}{c}433 \\
30^{\mathrm{a}}\end{array}$ & $\begin{array}{r}110 \\
5\end{array}$ & $\begin{array}{c}162 \\
15^{\mathrm{a}}\end{array}$ & $\begin{array}{r}81 \\
2\end{array}$ \\
\hline $\begin{array}{l}\text { No. de individuos } \\
\text { Campylobacter spp. (\%) } \\
\text { Shigella spp. (\%) }\end{array}$ & $\begin{array}{c}582 \\
17^{\mathrm{a}} \\
3\end{array}$ & $\begin{array}{r}229 \\
6 \\
<1\end{array}$ & $\begin{array}{r}537 \\
14 \\
5^{b}\end{array}$ & $\begin{array}{r}134 \\
17 \\
1\end{array}$ & $\begin{array}{r}433 \\
12 \\
4^{b}\end{array}$ & $\begin{array}{r}104 \\
13 \\
0\end{array}$ & $\begin{array}{c}307 \\
11^{\mathrm{a}} \\
4\end{array}$ & $\begin{array}{r}58 \\
3 \\
2\end{array}$ & $\begin{array}{c}483 \\
8 \\
11^{\mathrm{a}}\end{array}$ & $\begin{array}{r}128 \\
9 \\
2\end{array}$ & $\begin{array}{r}173 \\
5 \\
24^{\mathrm{a}}\end{array}$ & $\begin{array}{r}128 \\
5 \\
6\end{array}$ \\
\hline $\begin{array}{l}\text { No. de individuos } \\
\text { E. coli serogrupos O clásicos (\%) } \\
\text { E. coli enterotoxígena (\%) }\end{array}$ & $\begin{array}{r}144 \\
12^{\mathrm{a}} \\
4^{\mathrm{b}}\end{array}$ & $\begin{array}{r}109 \\
0 \\
0\end{array}$ & $\begin{array}{r}109 \\
12 \\
6\end{array}$ & $\begin{array}{r}50 \\
2 \\
0\end{array}$ & $\begin{array}{r}80 \\
9 \\
8\end{array}$ & $\begin{array}{r}21 \\
0 \\
0\end{array}$ & $\begin{array}{r}72 \\
6 \\
1\end{array}$ & $\begin{array}{r}16 \\
0 \\
0\end{array}$ & $\begin{array}{r}95 \\
5 \\
3\end{array}$ & $\begin{array}{r}31 \\
0 \\
0\end{array}$ & $\begin{array}{r}13 \\
8 \\
0\end{array}$ & $\begin{array}{r}14 \\
0 \\
0\end{array}$ \\
\hline
\end{tabular}

a $P<0,001$ (prueba exacta de Fisher).

b $P<0,05$ (prueba exacta de Fisher).

\section{Distribución de los agentes etiológicos según la edad}

Los rotavirus fueron significativamente más frecuentes en los casos que en los controles en todos los grupos de edad y constituyeron la principal causa de diarrea en niños de 3 a 24 meses de edad; su frecuencia fue baja en los casos de 0 a 2 meses $(16 \%)$ y empezó a aumentar a partir de los 3 meses, manteniéndose alta en el primer año de vida, para disminuir en el segundo y tercer años. Campylobacter spp. solo fue significativamente más frecuente en los casos que en los controles en el grupo de 0 a 2 meses ( $17 \%$, frente a $6 \%$ ); su frecuencia en los casos fue disminuyendo a medida que aumentaba la edad (de $17 \%$ en el grupo de 0 a 2 meses a $5 \%$ después de los 2 años). E. coli de los serogrupos O clásicos y E. coli enterotoxígena fueron importantes como agentes etiológicos solamente en los niños menores de 3 meses $(12 \%, P<0,001 ; y 4 \%$, $P<0,05$, respectivamente). Shigella spp. tuvo un comportamiento diferente al de los demás agentes, ya que su frecuencia mostró tendencia a aumentar con la edad, especialmente a partir de los 12 meses (cuadro 4).

\section{Manifestaciones clínicas}

El cuadro 5 muestra las características clínicas de los episodios de diarrea en los que se identificó un solo agente etiológico. Los rotavirus se asociaron de forma estadísticamente significativa con la deshidratación $(P<0,001)$ y los vómitos $(P<0,001)$, Shigella spp. con la fiebre $(P<0,05)$ y Campylobacter spp. y Shigella spp. con la mayor duración de los episodios de diarrea $(P<0,05)$ y la presencia de sangre en las heces $(P<0,001)$.

La frecuencia de episodios de diarrea, tanto en total como en asociación con deshidratación, disminuyó progresivamente con la edad (figura 1). Al comparar la frecuencia de deshidratación según el agente etiológico (rotavirus frente a bacterias), se observa que en el grupo de 0 a 2 meses esta compli- cación fue más frecuente en los niños infectados por bacterias, mientras que en el grupo de 3 a 5 meses fue más frecuente en los infectados por rotavirus. De los 3 a 5 meses en adelante, los rotavirus siguieron siendo más frecuentes que las bacterias como causa de deshidratación hasta los 18 meses de edad, cuando las dos curvas empezaron a acercarse (figura 2).

\section{DISCUSIÓN}

En este estudio, realizado en cuatro ciudades de Venezuela, las características de los niños (sexo, edad, estrato

CUADRO 5. Manifestaciones clínicas de la diarrea aguda según el agente etiológico.a Venezuela, 1993-1995

\begin{tabular}{|c|c|c|c|c|c|c|c|c|c|c|}
\hline \multirow[b]{3}{*}{ Característica clínica } & \multicolumn{10}{|c|}{ Agente etiológico } \\
\hline & \multicolumn{2}{|c|}{$\begin{array}{l}\text { Rotavirus } \\
(n=486)\end{array}$} & \multicolumn{2}{|c|}{$\begin{array}{c}\text { Campylobacter } \\
(n=150)\end{array}$} & \multicolumn{2}{|c|}{$\begin{array}{l}\text { Shigella } \\
(n=102)\end{array}$} & \multicolumn{2}{|c|}{$\begin{array}{l}\text { ECSOb }^{b} \\
(n=23)\end{array}$} & \multicolumn{2}{|c|}{$\begin{array}{l}\mathrm{ECET}^{\mathrm{c}} \\
(n=6)\end{array}$} \\
\hline & No. & $\%$ & No. & $\%$ & No. & $\%$ & No. & $\%$ & No. & $\%$ \\
\hline$>5$ evacuaciones/día & 364 & 75 & 111 & 74 & 82 & 80 & 12 & 52 & 5 & 83 \\
\hline Fiebre & 231 & 48 & 63 & 42 & 64 & $63^{e}$ & 6 & 26 & 1 & 17 \\
\hline Vómitos & 393 & $81^{d}$ & 62 & 41 & 56 & 5 & 10 & 44 & 1 & 17 \\
\hline Sangre en heces & 41 & 8 & 74 & $49^{d}$ & 60 & $59^{d}$ & 0 & 0 & 0 & 0 \\
\hline Diarrea > 5 días & 143 & 29 & 62 & $41^{\mathrm{e}}$ & 49 & $48^{e}$ & 5 & 22 & 1 & 17 \\
\hline Deshidratación & 280 & $58^{d}$ & 35 & 23 & 21 & 21 & 9 & 39 & 2 & 33 \\
\hline
\end{tabular}

a En casos con un único agente etiológico.

b E. coli serogrupos $O$ clásicos.

c E. coli enterotoxígena.

d $P<0,001$ (prueba exacta de Fisher).

e $P<0,05$ (pueba exacta de Fisher). 
FIGURA 1. Distribución de los episodios de diarrea aguda, totales y con deshidratación, según la edad. Venezuela, 1993-1995

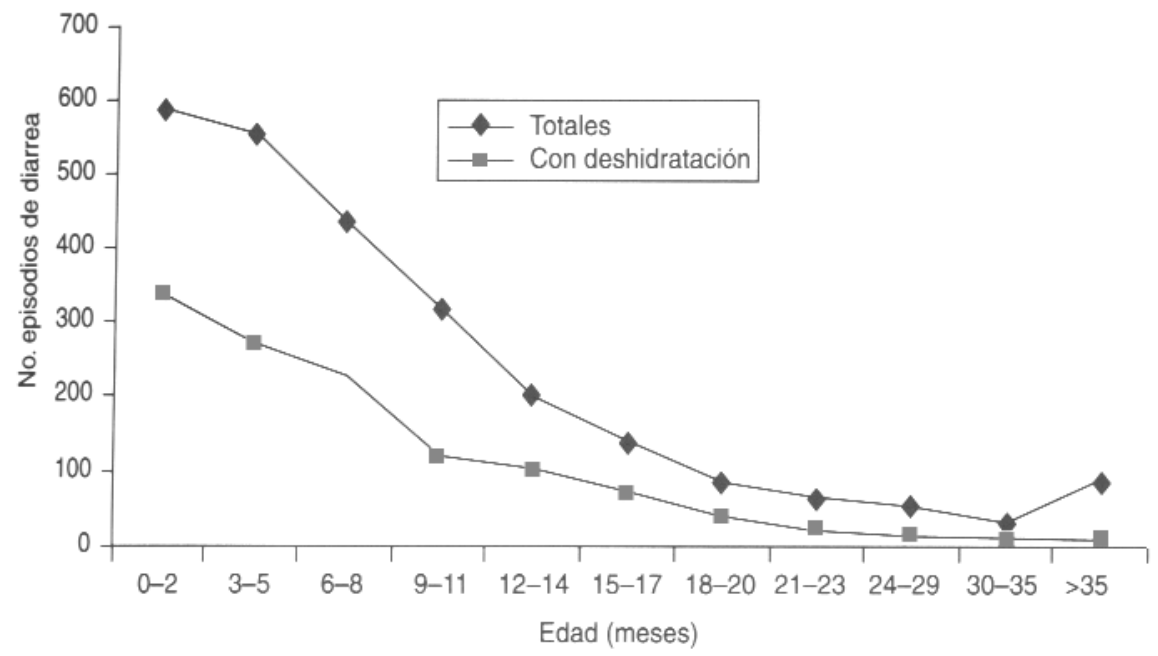

socioeconómico, estado nutricional y tipo de alimentación) fueron similares en las cuatro ciudades, con la excepción de Mérida, donde hubo mayor proporción de niños de clase socioeconómica más alta y de niños alimentados con leche materna. Además, el promedio de edad (11 meses) fue superior al de la totalidad de la población (6 meses) y hubo una mayor proporción de episodios durante el segundo año de vida, diferencias que podrían explicarse, al menos parcialmente, por la mayor frecuencia de la lactancia materna. Estos datos confirman el efecto protector de la lactancia materna, que retrasa la aparición de la diarrea hasta el segundo semestre de la vida $(21,22)$. Asimismo, se confirmó la asociación entre la diarrea y la desnutrición $(1,2)$.

Estos resultados muestran la importancia de los rotavirus como causa de diarrea y deshidratación durante los dos primeros años de vida. Estos datos, similares a los obtenidos en otras partes del mundo (23-26) y en otros estudios venezolanos $(5,27)$, de-

FIGURA 2. Distribución de los episodios de deshidratación según la edad y el agente etiológico. Venezuela, 1993-1995

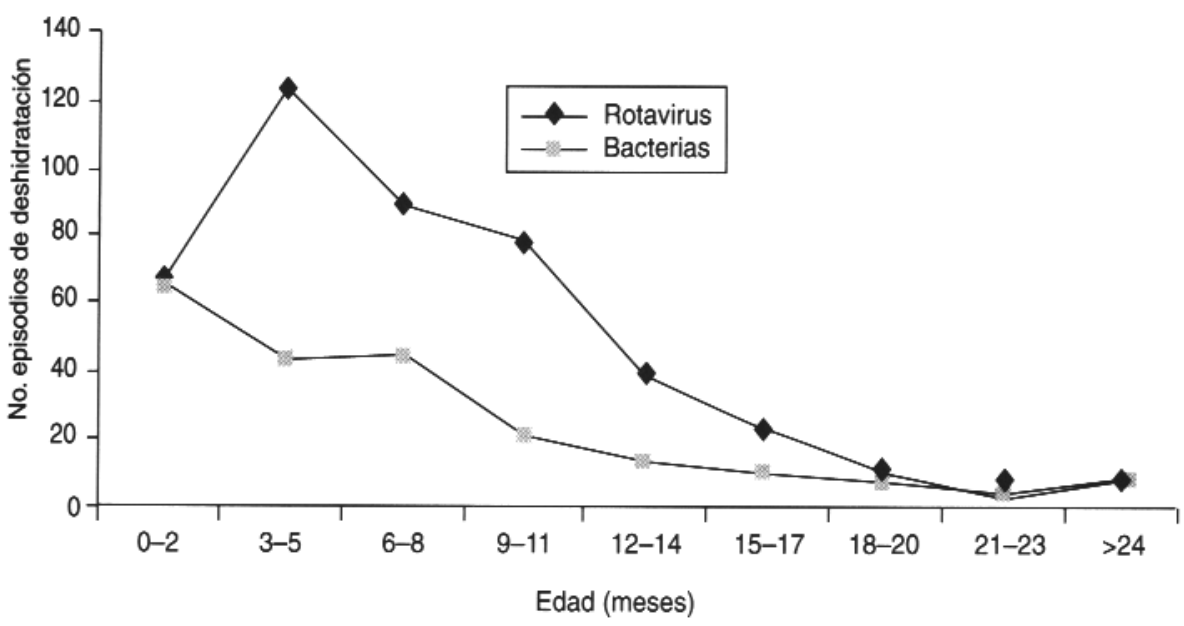

muestran una vez más que los rotavirus son la principal causa de diarrea atendida en el hospital y reafirman la necesidad de controlarlos. En consecuencia, una vacuna tendría gran impacto sobre los costos de atención hospitalaria $(5,8-10,28)$.

Estudios anteriores han mostrado la circulación en Venezuela de los cuatro serotipos $\mathrm{G}$ de rotavirus epidemiológicamente más importantes, generalmente con predominio de un serotipo sobre los demás (13). Sin embargo, en este estudio se detectó casi exclusivamente el serotipo G1, que representó casi $99 \%$ de las cepas tipificadas. En estudios realizados en otros países, como el Brasil y la India, se ha descrito la existencia de serotipos distintos de los más frecuentes, como el G5 y el G9 (28-30), pero en Venezuela no parece ser este el caso, ya que solamente se han encontrado tres tipos de patrones electroforéticos (Liprandi, datos no publicados), lo cual refleja un polimorfismo genético limitado y la circulación de pocas cepas virales. Esta circunstancia, sin explicación hasta ahora, es poco usual porque se ha demostrado que los rotavirus presentan una amplia diversidad genómica (31-33). El porcentaje de muestras no tipificables por ELISA fue similar al registrado en estudios anteriores $(13,29)$.

Campylobacter, el segundo agente más frecuente en este estudio, fue especialmente importante en los menores de 3 meses. A partir de esta edad no se observaron diferencias entre los pacientes y los controles. Esta situación es similar a la descrita en otros países en desarrollo $(24,26,34,35)$ y podría obedecer a la adquisición de inmunidad durante épocas tempranas de la vida, que probablemente protege contra la enfermedad sin impedir la aparición de reinfecciones, como se ha demostrado en estudios serológicos anteriores (36-39). Sin embargo, otros estudios no han detectado diferencias en la frecuencia de Campylobacter entre los pacientes y los controles (40-43), lo cual sugiere la existencia de portadores asintomáticos o la presencia de cepas no patógenas en zonas de alta endemia (36).

El papel de E. coli de los serogrupos O clásicos como agente de diarrea se ha 
puesto en duda, pero en un estudio anterior (44) hemos registrado una correlación significativa entre el patrón de adherencia localizada y la presencia de diarrea. Los serogrupos más frecuentes fueron: O55, O119, O26, O127, O114, O111, O126 (44). El comportamiento de E. coli de los serogrupos O clásicos, encontrados con una frecuencia significativa solo en el grupo de 0 a 2 meses, fue similar al de Campylobacter y probablemente también esté asociado a la adquisición de inmunidad $(44,45)$.

A diferencia de los demás agentes, Shigella fue significativamente más frecuente en los niños mayores de un año, circunstancia que también se ha descrito en otros estudios $(25,26,46)$.

Aunque algunos autores $(24,35,47)$ consideran que la Salmonella es un agente patógeno importante en niños, este estudio coincidió con otros $(23,25)$ que han destacado su escasa importancia como causa de diarrea aguda en niños pequeños.

$\mathrm{Al}$ igual que en la mayoría de los trabajos realizados en niños $(23,48,49)$, los parásitos no desempeñaron un papel importante en la etiología de la diarrea aguda. Giardia lamblia fue incluso más frecuente en los controles que en los pacientes, probablemente debido a que la técnica utilizada tiene mayor sensibilidad en la recuperación de los quistes en heces no diarreicas $(43,48)$.

Este trabajo demuestra que la edad es un importante factor determinante de la prevalencia y gravedad de la diarrea aguda, como ha sido demostrado en anteriores oportunidades (50). Los rotavirus y las bacterias presentaron marcadas diferencias en la relación entre gravedad y edad. Mientras que la mayor frecuencia de deshidratación por rotavirus ocurrió en el grupo de 3 a 5 meses, con las bacterias la mayor frecuencia se registró en los niños más pequeños (0 a 2 meses). Esto sugiere que los niños menores de 3 meses están protegidos por los anticuerpos transplacentarios específicos para rotavirus y que no ocurre lo mismo con las diarreas bacterianas $(14,44)$. La lactancia materna desempeña un papel muy importante en la protección contra las diarreas bacterianas $(21,22,51$, 52 ), mientras que en el caso de los rotavirus su papel es todavía controvertido $(21,53)$. Sin embargo, la baja frecuencia $(21 \%)$ de la lactancia materna en la población estudiada, con la excepción de Mérida, podría explicar también la alta frecuencia de deshidratación en los tres primeros meses de vida y la elevada proporción (48\%) de niños enfermos en los primeros seis meses de vida.

En general, la mortalidad y morbilidad por diarrea se presentan a edades más tempranas en los países en desarrollo $(3,4,54)$, como ocurrió en este estudio. El bajo nivel educacional, la baja frecuencia de la lactancia materna y la insalubridad ambiental contribuyen a ello (21), particularmente porque se asocian con las diarreas de etiología bacteriana $(21,25,55,56)$. Los programas específicos destinados a fomentar la lactancia materna y la apropiada nutrición en el destete, así como la educación sobre medidas higiénicas personales y la manipulación de alimentos, podrían tener gran impacto en el control de la enfermedad en la población infantil (21). Además, la restricción del uso de antibióticos a los casos muy específicos que lo requieran, como las diarreas por Campylobacter, podría contribuir a mejorar el tratamiento de la enfermedad.

Las características epidemiológicas de la diarrea aguda presentan una gran variabilidad regional, particularmente acentuada entre los países en desarrollo y desarrollados, porque están obviamente vinculadas con las condiciones de vida $(21,25,54-56)$. En consecuencia, los programas de prevención deben ser locales, estar basados en información científica y ser evaluados en términos de costos y beneficios para conseguir la mayor eficacia posible, sobre todo en países como el nuestro, con limitados recursos médicos, sociales y financieros.

Agradecimiento. Los autores agradecen al Consejo Nacional de Investigaciones Científicas y Tecnológicas (CONICIT) la financiación del estudio (Mega Proyecto Social No. RP-IV140031). Se extiende un agradecimiento especial a Beatriz Nieves (Coordinadora de Mérida), Jesús Bastardo (Coordinador de Cumaná), Tulia Hernández (Coordinadora de Puerto Ordaz), Hans Romer, Mariana Mariño, Ana María Rojas, Noris Serrano, Zaida Carvajal, Luisa Vizcaya, Antonio Maldonado y Anaibeth Nessi por su participación en los estudios clínicos y de laboratorio.

\section{REFERENCIAS}

1. Guerrant RL, Schorling JB, McAuliffe JF, De Souza MA. Diarrhea as a cause and effect of malnutrition: diarrhea prevents catch-up growth and malnutrition increases diarrhea frequency and duration. Am J Trop Med Hyg 1992;47(1):28-35.

2. Pérez-Schael I, Dehollain P, Pérez M, Daoud N, Soto I, González M, et al. Impacto de las enfermedades diarreicas en el estado nutricional del niño. An Ven Nutr 1988;1: 119-128.
3. Bern C, Martines J, De Zoisa I, Glass RI. The magnitude of the global problem of diarrhoeal disease: a ten-year update. Bull World Health Organ 1992;70(6):705-714.

4. Bern C, Glass RI. Impact of diarrheal disease worlwide. En: Kapikian AZ, ed. Viral infections of the gastrointestinal tract. 2. ${ }^{\text {a }}$ ed. New York: Marcel Dekker; 1994. pp. 1-26.

5. Pérez-Schael I. The impact of rotavirus disease in Venezuela. J Infect Dis 1996;174(Supl 1):S19-S21.
6. Venezuela, Ministerio de Sanidad y Asistencia Social. Principales causas de muerte en Venezuela, 1986-1990. Caracas: Dirección de Planificación y Estadística; 1994.

7. Venezuela, Ministerio de Sanidad y Asistencia Social. Bol Epidemiol Sem, No 52 . Caracas Dirección de Vigilancia Epidemiológica; 1998;49(3836):650.

8. Kapikian AZ, Hoshino Y, Chanock RM, Pérez-Schael I. Efficacy of a quadrivalent rhesus rotavirus-based human rotavirus vaccine 
aimed at preventing severe rotavirus diarrhea in infants and young children. J Infect Dis 1996;174(Supl 1):S65-S72.

9. Pérez-Schael I, Guntiñas MJ, Pérez M, Pagone V, Rojas AM, González R, et al. Efficacy of rhesus rotavirus-based quadrivalent vaccine in infants and young children in Venezuela. N Engl J Med 1997;337(17):1181-1187.

10. Glass RI, Bresee JS, Parashar U, Miller M, Gentsch JR. Rotavirus vaccines at the threshold. Nat Med 1997;3(12):1324-1325.

11. Méndez-Castellano H, De Méndez MC. Estratificación social y biología humana: método de Graffar modificado. Arch Ven Pueric Pediatr 1986;49(3/4):93-104.

12. Kapikian AZ, Yolken RH, Greenberg HB, Wyatt RG, Kalica AR, Chanock RM, et al. Gastroenteritis viruses. En: Lennette $\mathrm{EH}$, Schmidt NJ, eds. Diagnostic procedures for viral, rickettsial and chlamydial infections. 5. ${ }^{\mathrm{a}}$ ed. Washington, D.C.: American Public Health Association; 1979. pp. 927-995.

13. White L, García D, Boher Y, Blanco M, Pérez $\mathrm{M}$, Romer $\mathrm{H}$, et al. Temporal distribution of human rotavirus serotypes 1, 2, 3, and 4 in Venezuelan children with gastroenteritis during 1979-1989. J Med Virol 1991;34(2):79-84.

14. Rojas AM, Boher Y, Guntiñas MJ, PérezSchael I. Homotypic immune response to primary infection with rotavirus serotipe G1. J Med Virol 1995;47(4):404-409.

15. Lennette EH, Ballows A, Hauser Jr WJ, Shadomy HJ, eds. Manual of Clinical Microbiology. 4. ${ }^{\text {a }}$ ed. Washington, D.C.: American Society for Microbiology; 1985

16. Orskov F, Orskov I. Serotyping of Escherichia coli. En: Bergen E, ed. Methods in microbiology. London: Academic Press; 1984. pp. 43-112.

17. Kudoh Y. Detection of heat-labile enterotoxin of Escherichia coli by reversed passive hemagglutination test with specific immunoglobulin against cholera toxin. Proceedings of the $14^{\text {th }}$ Joint Conference, U.S.-Japan Cooperative Medical Science Program. Cholerae Panel, Toho University, Tokyo; 1979. pp. 266.

18. Thompson MR, Brandwein H, LaBine-Racke M, Gianella RA. Simple and reliable enzymelinked immunosorbent assay with monoclonal antibodies for detection of Escherichia coli heat-stable enterotoxins. J Clin Microbiol 1984;20(1):59-64.

19. Day NP, Scotland SM, Rowe B. Comparison of an HEp-2 tissue culture test with the Sereny test for detection of enteroinvasiveness in Shigella spp. and Escherichia coli . J Clin Microbiol 1981;13(3):596-597.

20. Pérez de Suárez E, Nessi A, Pérez-Schael I, Mariño M, Niño E, Romer H, et al. Blastocystis hominis en niños con gastroenteritis estudiados en dos centros hospitalarios pediátricos de Caracas, Venezuela, junio 1993-julio 1994. Arch Ven Puer Pediatr 1997;60(4):167-170.

21. Huttly SRA, Morris SS, Pisani V. Prevention of diarrhoea in young children in developing countries. Bull World Health Organ 1997; 75(2):163-174

22. Glass RI, Stoll BJ. The protective effect of human milk against diarrhea. Acta Paediatr Scand 1989; 351:131-136

23. Black RE, Merson MH, Rahman AS, Yunus M, Alim AR, Huq I, et al. A two-year study of bacterial, viral, and parasitic agents associated with diarrhea in rural Bangladesh. J Infect Dis 1980;142(5):660-664.

24. Echeverría P, Taylor DN, Lexsomboon U, Bhaibulaya M, Blacklow NR, Tamura K, et al. Case-control study of endemic diarrheal disease in Thai children. J Infect Dis 1989;159(3): 543-548.

25. Seigel RR, Santana C, Salgado K, De Jesus P. Acute diarrhea among children from high and low socioeconomic communities in Salvador, Brazil. Int J Infect Dis 1996;1(1):28-34.

26. Huilan S, Zhen LG, Mathan MM, Mathew MM, Olarte J, Espejo R, et al. Etiology of acute diarrhoea in developing countries: a multicentre study in five countries. Bull World Health Organ 1991;69(5):549-555.

27. Pérez-Schael I, Rojas AM, Flores J. Desarrollo de una vacuna anti-rotavirus. Pruebas de campo en Venezuela. Acta Cient Venez 1991; 42(6):296-312

28. Bresse JS, Glass R I, Ivanoff B, Gentsch J R. Current status and future priorities for rotavirus vaccine development, evaluation, and implementation in developing countries. Vaccine 1999; 17:2207-2222.

29. Gentsch RJ, Woods PA, Ramachandran M, Das BK, Leite JP, Alfieri A, et al. Review of G and $P$ typing results from a global collection of rotavirus strains: implications for vaccine development. J Infect Dis 1996;174(Suppl 1): 30-36.

30. Timenetsky MC, Santos N, Gouvea V. Survey of rotavirus $\mathrm{G}$ and $\mathrm{P}$ types associated with human gastroenteritis in São Paulo, Brazil, from 1986 to 1992. J Clin Microbiol 1994; 32(10):2622-2624

31. Estes MK, Cohen J. Rotavirus gene structure and function. Microbiol Rev 1989;53(4): 410-449.

32. Kapikian AZ, Chanock RM. Rotaviruses. En: Fields BN, Knipe DM, Chanock RM, Hirsch MS, Melnick JL, Monath TP, et al., eds. Virology. New York: Raven Press; 1990. pp. 1353-1404.

33. Taniguchi $K$, Urasawa S. Diversity in rotavirus genomes. Virology 1995;6:123-131

34. Black RE, López de Romaña G, Brown KH, Bravo N, Bazalar OG, Kanashiro HC. Incidence and etiology of infantile diarrhea and major routes of transmission in Huascar Peru. Am J Epidemiol 1989;129(4):785-799.

35. Varavithya W, Vathanophas K, Bodhidatta L, Punyaratabandhu P, Sangchai R, Athipanyakom S, et al. Importance of Salmonellae and Campylobacter jejuni in the etiology of diarrheal disease among children less than 5 years of age in a community in Bangkok, Thailand. J Clin Microbiol 1990;28(11):2507-2510.

36. Taylor DN. Campylobacter infections in developing countries. En: Nachamkin I, Blaser MJ, Tompkins LS, eds. Campylobacter jejuni: current status and future trends. Washington, D.C.: American Society for Microbiology; 1992. pp. 20-30

37. Blaser MJ, Black DJ, Duncan DJ, Amer J. Campylobacter jejuni-specific serum antibodies are elevated in healthy Bangladeshi children. J Clin Microbiol 1985;21(2):164-167.

38. Blaser MJ, Taylor DN, Echeverría P. Immune response to Campylobacter jejuni in a rural community in Thailand. J Infect Dis 1986; 153(2):249-254
39. Martin PM, Mathiot J, Ipero J, Kirimat M, Georges AJ, Georges-Courbot MC. Immune response to Campylobacter jejuni and Campylobacter coli in a cohort of children from birth to 2 years of age. Infect Immun 1989;57(8): 2542-2546.

40. Georges MC, Wachsmuth IK, Meunier DMV, Nebout N, Didier F, Siopathis MR, et al. Parasitic, bacterial, and viral enteric pathogens associated with diarrhea in the Central African Republic. J Clin Microbiol 1984;19(5):571-575.

41. Georges-Courbot MC, Beraud-Cassel AM, Gouandjika I, Georges AJ. Prospective study of enteric Campylobacter infections in children from birth to 6 months in Central Africa Republic. J Clin Microbiol 1987;25(5):836-839.

42. Glass RI, Stoll BJ, Huq MI, Struelens MJ, Blaser M, Kibriya AK. Epidemiologic and clinical features of endemic Campylobacter jejuni infection in Bangladesh. J Infect Dis 1983; 148(2):292-296

43. Zaki AM, DuPont HL, El Alamy MA, Arafat RR, Amin K, Awad MM, et al. The detection of enteropathogens in acute diarrhea in a family cohort population in rural Egypt. Am J Trop Med Hyg 1986;35(5):1013-1022.

44. González R, Díaz C, Marino M, Cloralt R, Pequeneze M, Pérez-Schael I. Age-specific prevalence of Escherichia coli with localized and aggregative adherence in Venezuelan infants with acute diarrhea. J Clin Microbiol 1997;35(5):1103-1107.

45. Barros HC, Ramos SR, Trabulsi LR, Silva ML. Inhibition of enteropathogenic Escherichia coli adhesion to HeLa cells by serum of infants with diarrhea and by cord serum. Braz J Med Biol Res 1995;28(1):83-87.

46. Mathew M, Mathan MM, Mani K, George R, Jebakumar K, Dharamsi R, et al. The relationship of microbial pathogens to acute infectious diarrhoea of childhood. J Trop Med Hyg 1991;94(4):253-260.

47. Gomes TAT, Rassi V, MacDonald KL, Ramos SR, Trabulsi LR, Vieira MAM, et al. Enteropathogens associated with acute diarrheal disease in urban infants in São Paulo, Brazil. J Infect Dis 1991;164(2):331-337.

48. Molbak K, Wested N, Holyng N, Scheutz F, Gottschau A, Aaby P, et al. The etiology of early childhood diarrhea: a community study from Guinea-Bissau. J Infect Dis 1994;169(3): 581-587.

49. Ogunsanya TI, Rotimi VO, Adenuga A. A study of the aetiological agents of childhood diarrhoea in Lagos, Nigeria. J Med Microbiol 1994;40(1):10-14.

50. Victora CG, Fuchs SC, Kirkwood BR, Lombardi C, Barros FC. Breast-feeding, nutritional status, and other prognostic factors for dehydration among young children with diarrhoea in Brazil. Bull World Health Organ 1992; 70(4):467-475.

51. Blake PA, Ramos S, MacDonald KL, Rassi V, Gomes TA, Ivey C, et al. Pathogen-specific risk factors and protective factors for acute diarrheal disease in urban Brazilian infants. J Infect Dis 1993;167(3):627-632.

52. Ruiz-Palacios GM, Calva JJ, Pickering LK, López-Vidal Y, Volkow P, Pezzarossi H, et al. Protection of breast-fed infants against Campylobacter diarrhea by antibodies in human milk. J Pediatr 1990;116(5):707-713. 
53. Glass RI, Stoll BJ, Wyatt RG, Hoshino Y, Banu $\mathrm{H}$, Kapikian AZ. Observations questioning a protective role for breast-feeding in severe rotavirus diarrhea. Acta Paediatr Scand 1986; 75(5):713-718.

54. Murray CJL, López AD. Global and regional cause-of-death patterns in 1990. Bull World Health Organ 1994;72(3):447-480.
55. Levine MM, Levine OS. Changes in human ecology and behavior in relation to the emergence of diarrheal diseases, including cholera. Proc Natl Acad Sci USA 1994;91(7): 2390-2394.

56. Savarino SJ, Bourgeois AL. Diarrhoeal disease: current concepts and future challenges. Epidemiology of diarrhoeal diseases in devel- oped countries. Trans R Soc Trop Med Hyg 1993;87(Supl 3):7-11.

Manuscrito recibido el 30 de junio de 1998 y aceptado para publicación, tras revisión, el 15 de abril de 1999.

ABSTRACT In four cities of Venezuela a study was carried out to evaluate the epidemiological, clinical, and etiological characteristics of acute diarrhea in children under 5 years of age. The study was done between June 1993 and May 1995 and involved children who

Etiological, clinical, and sociodemographic characteristics of acute diarrhea in Venezuela were seen in a hospital, 2552 with diarrhea and 793 controls. The Fisher exact test was used for the statistical analysis of the results. Rotaviruses were the most important agents, both in terms of their frequency $(30 \%)$ and their association with dehydration $(58 \%)$. Following in importance were Campylobacter spp. (13\%) and Escherichia coli classical O serogroups (9\%), but their association with diarrhea was only statistically significant among children less than 3 months old, a fact that is particularly important from the standpoint of treatment. The importance of age was confirmed as a determining factor in the prevalence and severity of diarrhea. 